\title{
Analysis of Steady State Creep in Short Fiber Composites (SFC) by Polynomial Function (PF) and Boundary Condition Method (BCM)
}

\author{
Vahid Monfared \\ Department of Mechanical Engineering, Zanjan Branch, Islamic Azad University, Zanjan, Iran
}

\begin{abstract}
Two novel mathematical models based on polynomial function (PF) and boundary condition method (BCM) are presented for determination of shear stress in interface in steady state creep in short fiber composites under axial loading. Important novelty of this research is determination of shear stress in interface without using of shear lag and imaginary fiber technique (IFT) theories. In these approaches, determination of shear stress in interface by polynomial function (PF) and boundary condition method (BCM)are easier and more comfortable than the previous analytical methods, . Determination of shear stresses in interface in steady state creep in short fiber composites are very important for design of composites. Analysis of shear stress in interface are used for investigation of failure, fracture and fatigue in short fiber composites in steady state creep. Finally, good agreements are found between these two methods and previous analytical and numerical predictions for behaviour of steady state creep in short fiber composites.
\end{abstract}

Keywords Composites, Steady State Creep, polynomial function (PF), Boundary condition method (BCM), Numerical Predictions

\section{Introduction}

Creep is more critical and important in materials that are subjected to high temperatures and loads for long periods, and close to melting point. The increasing application of short fiber composites (SFC) in high loads and temperatures environments requires a thorough knowledge of their creep characteristics and deformation mechanisms. Numerous investigators have studied the steady state creep behaviour by analytical and experimental methods. Most of them have investigated second stage creep problems by analytical, experimental and numerical methods (FEM) by using of famous mechanics theories. Important novelty of this new work is determination of shear stress in interface without using of famous mechanics theories such as shear lag and imaginary fiber technique (IFT) theories. Also, shear stress in interface in second stage creep in short fiber composites is determined directly by polynomial function (PF) and boundary condition method (BCM). New work results are compared with previous analytical and numerical predictions for behaviour of steady state creep behaviour in short fiber composites. Eventually, excellent agreements are found between these new methods and previous analytical and

* Corresponding author:

vahid_monfared_57@yahoo.com (Vahid Monfared)

Published online at http://journal.sapub.org/mechanics

Copyright (C) 2012 Scientific \& Academic Publishing. All Rights Reserved numerical predictions for behaviour of steady state creep in short fiber composites. Shear stress in interface in steady state creep in short fiber composites is significant and consequential for composites analysis and design. Determination of shear stress values in interface (fiber and matrix) are used for analysis of failure, fracture and fatigue in short fiber composites in steady state creep. In recent times, comprehensive studies have been performed to determine the creep behaviour of short fiber composites in second stage creep [1-6]. Also, in the present new research, analytical and mathematical approaches for prediction of the steady state creep behaviour of short fiber composites based on polynomial function (PF) and boundary condition method (BCM) are proposed without using of some theories. Here, an axisymmetric unit cell representing a fiber with its surrounding matrix as two coaxial cylinders is considered. For verification of the solution method, the SiC/6061Al composite is selected as a case study and the results will be compared with the previous experimental available results in [5]. New mathematical formulation based on polynomial function (PF) and boundary condition method (BCM) are presented for determination of exact second stage creep behaviour in short fiber metal matrix composites 6061Al $115 \% \mathrm{SiC}$ (MMC's) under axial loading. Polynomial function method (PFM or PF) and boundary condition method (BCM) are precise, meticulous and extremely accurate approach for determination of interface shear stress results in MMC's. In addition polynomial function is presented analytically. As 
well as, shear stress in interface of unit cell is determined by two independent methods. These polynomial functions are functions of material creep properties, geometric factors and applied stress.

Mondali et al. [1] proposed new analytical shear-lag based model for prediction of the steady state creep deformations of some short fiber composites by Imaginary fiber technique. Boubakri et al. investigated influence of thermal aging on tensile and creep behavior of thermoplastic polyurethane [2]. Creep of fibrous composite materials has been studied by Lilholt [3]. FEM study of the second stage creep behavior of Al6061/SiC metal matrix composite has been studied by Mondali et al. [4]. Morimoto et al. [5] investigated second stage creep of silicon carbide whisker/6061 aluminum composite at $573 \mathrm{~K}$. Ghavami et al. presented finite difference solution of steady state creep deformations in a short fiber composite in presence of fiber/matrix debonding [6]. Monfared [7] proposed new analytical formulation for contact stress and prediction of crack propagation path in rolling bodies and comparing with finite element model (FEM) results statically. Prediction of Mechanical Behavior of PZT and SMA have been studied by Monfared and Khalili [8]. Cibulka presented constrained open mapping theorem with applications [9]. Gutknecht proposed numerical conformal mapping methods based on function conjugation [10].

Mishra and Pandey [11] investigated some observations on the high-temperature creep behaviour of $6061 \mathrm{Al}-\mathrm{SiC}$ composites. Boniecki, et al. [12] studied high temperature deformation of ceramic particle composites. Steady state creep of a composite with short fibres was investigated by Mileiko [13]. Fukuda and Chou proposed advanced shear-lag model applicable to discontinuous fiber composites [14]. Xu et al. [15] investigated high temperature simulation of short carbon fiber-reinforced nickel base composite. Nedjar presented time dependent model for unidirectional fibre-reinforced composites with viscoelastic matrices [16]. Nieh [17] studied creep rupture of a silicon-carbide reinforced aluminium composite. Creep deformation of metal-matrix composites have been investigated by McLean [18]. Analysis of the effect of temperature on the creep parameters of composite material was investigated by Kouadri-Boudjelthia et al. [19].

Pachalis et al. [20] presented modelling of creep of aligned short-fiber reinforced ceramic composites. Wang and Chou proposed analytical modelling of creep of short fiber reinforced ceramic matrix composite [21]. The elasticity and strength of paper and other fibrous materials has been investigated by Cox [22]. Interfacial debonding and fiber pull-out stresses of fiber-reinforced composites has been studied by Hsueh [23]. Interfacial debonding and fiber pullout stresses of fiber-reinforced composites Part VII: Improved analysis for bonded interfaces was investigated by Hsueh [24].

A modified analysis for stress transfer in fiber-reinforced composites with bonded fiber ends was proposed by Hsueh [25]. Hsueh and Becher studied residual thermal stresses in ceramic composites [26]. Hsueh et al. [27] investigated stress transfer in a model composite containing a single embedded fiber. Li and Langdon [28] presented examination of creep data for an Al-Mg composite.

$\mathrm{Gao}$ and $\mathrm{Li}$ [29] presented shear-lag for carbon nanotube-reinforced polymer composites. Jiang et al. [30] presented analytical model for elastic stress field distribution in fibre composite with partially debonded interface.

Lee et al. [31] proposed direct numerical predictions for the steady-state creep deformation of extruded SiCw/Al6061 composites using a representative volume element with random arrangement of whiskers.

\section{Material and Methods}

The cylindrical unit cell depicted in Figure 1 has been used to model a short fiber composite. In the mentioned model supposed that a cylindrical fiber with a radius a and a length $2 \mathrm{~L}$ is embedded in a coaxial cylindrical matrix with an outer radius $b$ and a length $2 \mathrm{~L}^{\prime}$. The volume fraction and aspect ratio of the fiber are defined as $\mathrm{f}$ and $\mathrm{s}=\mathrm{L} / a$ respectively. Also, in this work, $\mathrm{k}=\left(\mathrm{L}^{\prime} / \mathrm{b}\right) /(\mathrm{L} / \mathrm{a})=\mathrm{L}^{\prime} \mathrm{a} / \mathrm{Lb}$ is considered as a parameter related to the geometry of the unit cell. An applied axial stress $\sigma_{\text {app }}$ is evenly induced on the end faces of the unit cell (at $z= \pm L^{\prime}$ ). Steady state condition of stress is assumed and a full and perfect fiber-matrix interface is considered. Elastic deformations are very small and are neglected as compared to creep deformations. The fibers have elastic behaviour during the analysis and the Steady state creep behaviour of the matrix, which its properties are considered to be constant with temperature, is described by an exponential law as given in Equation 1,

$$
\dot{\varepsilon_{\mathrm{e}}}=\operatorname{Aexp}\left(\sigma_{\mathrm{e}} / \mathrm{B}\right)
$$

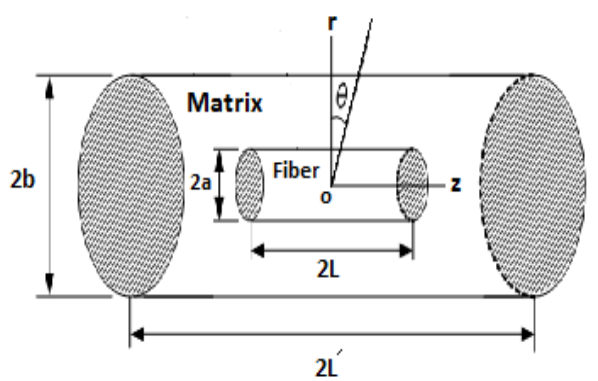

Figure 1. Unit cell model

Also $\dot{\varepsilon}_{\mathrm{e}}$ and $\sigma_{\mathrm{e}}$ are the equivalent stress and equivalent strain rate of the matrix respectively, will be functions of $r$ and $\mathrm{z}$ coordinates. Thus, it turns out to be a very complex nonlinear problem. For validation and verification of the solution method, the $\mathrm{SiC} / 6061 \mathrm{Al}$ composite is selected as a case study and the results will be compared with the previous experimental and analytical results. For the composite used here ( $\mathrm{SiC} / \mathrm{Al} 6061)$, the volume fraction (f) of fibers is $15 \%$ and the fibers have an aspect ratio (s) of 7.4 and $\mathrm{k}=0.76[5]$. Also, the steady state creep constants of the matrix material, $\mathrm{A}$ and $\mathrm{B}$, in Equation 1 are considered as $\mathrm{A}=\exp (-24.7)$ and $\mathrm{B}=6.47[5]$. In this section, boundary condition method $(\mathrm{BCM})$ is presented for determination of shear stress in in- 
terface in steady state creep behaviour in MMC's (Equations 2-11). This boundary condition method (BCM) is function of geometric factors, material properties and applied stress. This BCM is given by below relations,

$$
\begin{gathered}
\tau_{i}=\mathrm{f}\left(\mathrm{z}, \mathrm{l}, \mathrm{l}^{\prime}, \mathrm{B}, \mathrm{A}, \mathrm{s}, \mathrm{k}, \mathrm{f}, \mathrm{z}, \sigma_{\mathrm{app}}\right) \\
\tau_{i} \propto \mathrm{z}^{\alpha} \times \mathrm{l}^{\beta} \mathrm{l}^{\gamma} \times \mathrm{A}^{\delta} \times \mathrm{B}^{\varepsilon} \times \sigma_{\mathrm{app}}^{\epsilon} \times \mathrm{f}^{\vartheta} \mathrm{s}^{\theta} \mathrm{k}^{\mu} \times \mathrm{\aleph} \\
\tau_{i}\left(\frac{z}{l}\right)=c_{1} \operatorname{Ln}\left(1+\frac{z}{l}\right)+c_{2}
\end{gathered}
$$

And boundary conditions for this problem are given as,

$$
\begin{gathered}
\tau_{i}(0)=0 \\
\tau_{i(\max )}=\tau_{i}(1)=\rho \operatorname{Ln}(\varphi)
\end{gathered}
$$

Where have,

$$
\begin{gathered}
\varphi=\frac{1}{\operatorname{Aexp}(-\psi)} \\
\Psi=B \times\left(\mathrm{l}-\mathrm{l}^{\prime}\right) \mathrm{f}^{4} \mathrm{~s}^{1} \mathrm{k}^{3} \sigma_{\mathrm{app}}^{1} \\
\tau_{i(\max )} \propto\left(\frac{z}{l}\right)^{h} \times \operatorname{Ln}(\varphi) \times k^{\prime} \\
c_{1}=\frac{\rho \operatorname{Ln}(\varphi)}{\operatorname{Ln}(2)} \\
c_{2}=0
\end{gathered}
$$

Now, other method based on polynomial functions (PF) are introduced (Equations 12,13), that is,

$$
\begin{gathered}
\tau_{i}\left(\frac{z}{l}\right)=\sum_{j=0}^{n} \Omega_{j}\left(\frac{z}{l}\right)^{j} \\
\Omega_{j}=\Omega\left(\mathrm{z}, \mathrm{l}, \mathrm{l}^{\prime}, \mathrm{B}, \mathrm{A}, \mathrm{s}, \mathrm{k}, \mathrm{f}, \mathrm{z}, \sigma_{\mathrm{app}}\right)
\end{gathered}
$$

Where unknowns $\aleph, \delta, \emptyset, \omega, \alpha, \beta, \gamma, \varepsilon, \epsilon, \theta, \vartheta$ are obtained by analytical, finite element analysis (FEA) and experimental results, these results given as, $\varepsilon=\epsilon=1, \delta=$ $-1, \alpha=1, \beta=\gamma=\theta=1, \vartheta=4, \mu=3, N=1$.

Also, A, B are material properties in steady state creep in MMC's. Also, shear stress in interface i determined without using of some complex and intricate theories such as shear lag and imaginary fiber technique (IFT). This new approach is very easy, uncomplicated and direct.

\section{Results and Discussion}

Polynomial function $(\mathrm{PF})$ and boundary condition method (BCM) were proposed for analyzing interfacial shear stress in steady state creep in short fiber composites (CMC's). These polynomial functions (PF) and Boundary condition method (BCM) are based on material properties, geometric factors (unit cell) and applied stress in second stage of creep. These analyses show that polynomial functions (PF) and Boundary condition method are very exact for accurate determination of steady state creep behaviour. That is, polynomial function and boundary condition method determine shear stress values in interface exactly. These discussions have been shown in Figures 2, 3, 4 and Table 1. These figures and tables show that the boundary condition method $(\mathrm{BCM})$ and polynomial function (PF) results are closed to the previous analytical and numerical researches such as reference[1]. In addition, in this work, calculation of some results is simpler than the previous analytical results such as shear stress in interface by boundary condition method $(\mathrm{BCM})$ and polynomial function (PF). In addition, in this research, calculation of results is simpler than the previous analytical results such as shear stress in interface by bound- ary condition method and polynomial function (PF).

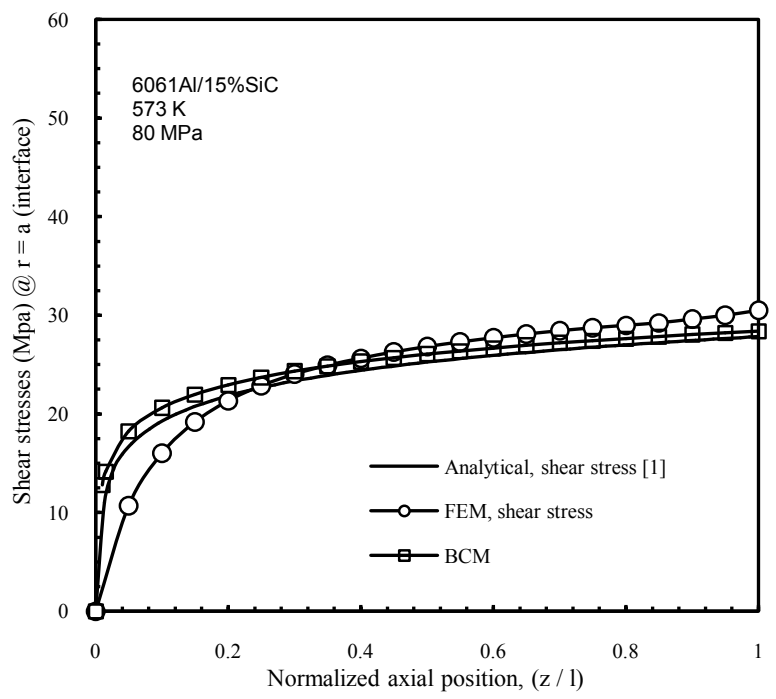

Figure 2. Analytical (BCM, [1]) and FEM curves of shear stresses at the interface

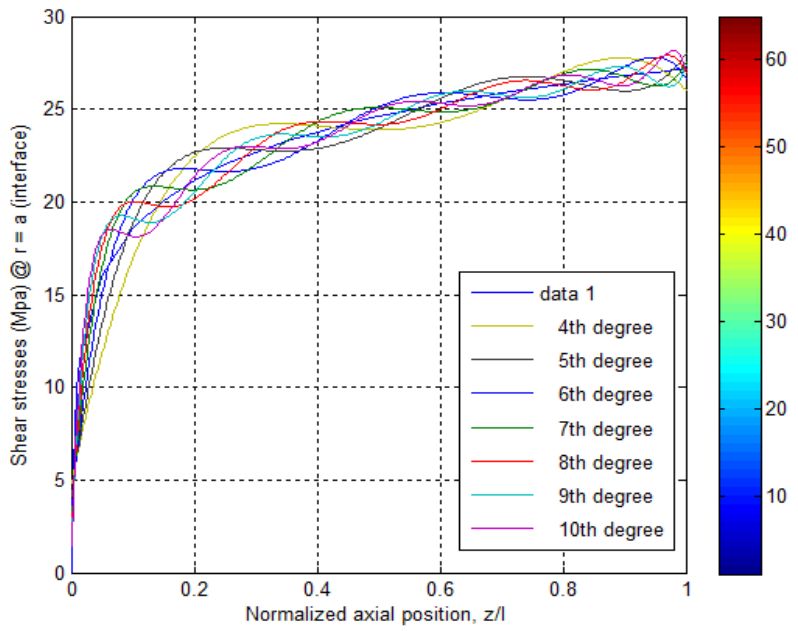

Figure 3. Polynomial function (PF) results for shear stresses at the interface

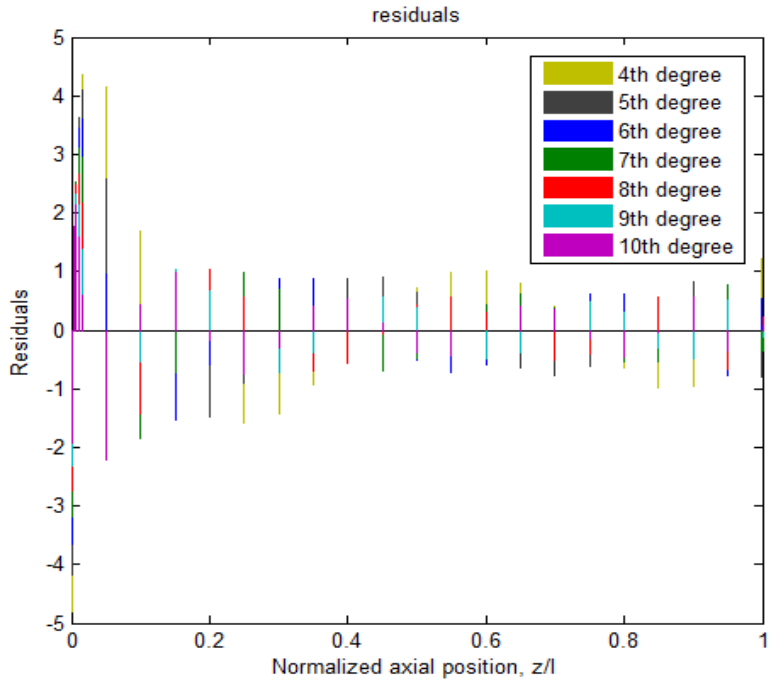

Figure 4. Residual of Polynomial functions (PF) results for interface shear stress 
Vahid Monfared: Analysis of Steady State Creep in Short Fiber Composites (SFC) by

Polynomial Function (PF) and Boundary Condition Method (BCM)

Table 1. Comparison of four approaches (FEM, BCM, PF and ref[1]) results for determination of shear stress in interface

\begin{tabular}{|c|c|c|c|c|c|c|c|c|c|c|c|}
\hline \multirow{2}{*}{ Shear stress (Interface, MPa) } & \multicolumn{11}{|c|}{ Normalized axial position, z/l } \\
\hline & 0 & 0.1 & 0.2 & 0.3 & 0.4 & 0.5 & 0.6 & 0.7 & 0.8 & 0.9 & 1 \\
\hline Ref [1], analytical & 0 & 19.53 & 21.87 & 23.3 & 24.45 & 25.24 & 25.97 & 26.51 & 26.99 & 27.45 & 27.82 \\
\hline FEM & 0 & 15.996 & 21.34 & 24.018 & 25.649 & 26.805 & 27.703 & 28.424 & 29.002 & 29.6 & 30.5 \\
\hline BCM (Newwork) & 0 & 20.59 & 22.944 & 24.31 & 25.293 & 26.05 & 26.668 & 27.19 & 27.643 & 28.04 & 28.4 \\
\hline PF(New work), $n=10$ & 0 & 20.8 & 20.12 & 24.87 & 22.52 & 27.2 & 23.56 & 28.23 & 24.9 & 29.1 & 26.93 \\
\hline
\end{tabular}

Table 1 shows comparison of four methods (FEM, BCM, $\mathrm{PF}$ and ref [1]) results for obtaining of shear stress values in interface. For comparison aim, the finite element numerical calculations of creep behaviour of this short fiber composite are also done using the finite element commercial code of ANSYS (The axisymmetry method with non-linear quadratic element of plane182 is employed for finite element analysis (FEM or FEA). This element is a higher order eight-node element and has creep modelling ability). According to Equation 12 typical form of the PF (Polynomial function) used in this work is in order $10(n=10)$.

\section{Conclusions}

Analytical formulations based on polynomial function (PF) and boundary condition method (BCM) were presented for determination of exact steady state creep behaviour in short fiber metal matrix composites $6061 \mathrm{Al} / 15 \% \mathrm{SiC}$ (MMC's) under axial tensile load. Polynomial function (PF) in steady state creep was presented in order to determine analytical shear stress in interface in fiber correctly. Also shear stress in interface was determined by boundary condition method (BCM) which their results were similar to the previous analytical and FEM results. One of the Advantages of these approaches is their simplicity and accuracy. Polynomial function method (PFM or PF) and boundary condition method (BCM) were exact approach for obtaining of interface shear stress results in second stage creep in MMC's.

Hence, Polynomial functions and boundary condition method (BCM) was presented analytically. Eventually, good agreement was found among polynomial function method $(\mathrm{PF})$, boundary condition method (BCM) results, previous analytical and FEM results for prediction of steady state creep behaviour in short fiber composites (SFC's). The mentioned methods (PFM, BCM) shown that the boundary condition method (BCM) and polynomial function (PF) results were closed to the previous analytical and numerical researches such as reference [1].

In addition, in this research, results were more precise than the previous analytical results such as shear stress in interface by boundary condition method and polynomial method. In addition, in these approaches, determination of results was easier than the previous analytical results such as determination of shear stress by shear lag or imaginary fiber technique.

\section{REFERENCES}

[1] Mondali, M., Abedian, A., Ghavami, A., 2009, A new analytical shear-lag based model for prediction of the steady state creep deformations of some short fiber composites, Mater. Design., 30, 1075-1084.

[2] Boubakri, A., Haddar, N., Elleuch, K., Bienvenu, Y., 2011, Influence of thermal aging on tensile and creep behavior of thermoplastic polyurethane, C. R. Mecanique., 339 (10), 666-673.

[3] Lilholt, H., 1985, Creep of fibrous composite materials, Compos. Sci. Technol., 22, 277-294.

[4] Mondali, A., Abedian, A., Adibnazari, S., 2005, FEM study of the second stage creep behavior of Al6061/SiC metal matrix composite, Comput. Mater. Sci., 34, 140-150.

[5] Morimoto, T., Yamaoka, T., Lilholt, H., Taya, M., 1988, Second stage creep of silicon carbide whisker/6061 aluminum composite at 573 K, J. Eng. Mater. Technol., 110, 70-76.

[6] Ghavami, A., Abedian , A., Mondali, M., 2010, Finite difference solution of steady state creep deformations in a short fiber composite in presence of fiber/matrix debonding, Mater. Design., 31, 2616-2624.

[7] Monfared, V., 2011, A new analytical formulation for contact stress and prediction of crack propagation path in rolling bodies and comparing with finite element model (FEM) results statically, Int. J. Phys. Sci., 6(15), 3613-3618.

[8] Monfared, V., Khalili, M.R., 2011, Investigation of Relations between Atomic Number and Composition Weight Ratio in PZT and SMA and Prediction of Mechanical Behavior, Acta phys. Pol. A., 120, 424-428.

[9] Cibulka, R., 2011, Constrained open mapping theorem with applications, J. Math. Anal. Appl., 379, 205-215.

[10] Gutknecht, M.H., 1986, Numerical conformal mapping methods based on function conjugation, J. Comput. Appl. Math., 14(1-2), 31-77.

[11] Mishra, R.S., Pandey, A.B., 1990, Some observations on the high-temperature creep behavior of $6061 \mathrm{Al}-\mathrm{SiC}$ composites, Metall. Mater. Trans. A., 21 (7), 2089-2090.

[12] Boniecki, M., Librant, Z., Wesołowski, W., 2012, High temperature deformation of ceramic particle composites, J. Eur. Ceram. Soc., 32 (1), 1-8.

[13] Mileiko, S.T., 1970, Steady state creep of a composite with short fibres, J. Mater. Sci., 5, 254-261.

[14] Fukuda, H., Chou, T.W., 1981, An advanced shear-lag model applicable to discontinuous fiber composites, J. Compos. Mater., 1(15), 79-91. 
[15] Xu, J., Zhao, L., Deng, X.,Yu, H., 2006, High temperature simulation of short carbon fiber-reinforced nickel base composite, Mater. Design., 27(10), 1152-1156.

[16] Nedjar, B., 2011, A time dependent model for unidirectional fibre-reinforced composites with viscoelastic matrices, Int. J. Solids. Struct., 48 (16-17), 2333-2339.

[17] Nieh, T.G., 1984, Creep rupture of a silicon-carbide reinforced aluminum composite, Metall. Trans. A., 15, 139-146.

[18] McLean, M., 1985, Creep deformation of metal-matrix composites, Compos. Sci. Technol., 23, 37-52.

[19] Kouadri-Boudjelthia, A., Imad, A., Bouabdallah, A., Elmeguenni, M., 2009, Analysis of the effect of temperature on the creep parameters of composite material, Mater. Design., 30(5), 1569-1574.

[20] Pachalis, J.R., Kim, J., Chou, T.W., 1990, Modeling of creep of aligned short-fiber reinforced ceramic composites, Compos. Sci. Technol., 37, 329-346.

[21] Wang, Y.R., Chou, T.W., 1992, Analytical modeling of creep of short fiber reinforced ceramic matrix composite, J. Compos. Mater., 26(9), 1269-1286.

[22] Cox, H.L., 1952, The elasticity and strength of paper and other fibrous materials, Br. J. Appl. Phys., 3, 72-79.

[23] Hsueh, C.H., 1990, Interfacial debonding and fiber pull-out stresses of fiber-reinforced composites, Mater. Sci. Eng., A123(1), 1-11.

[24] Hsueh, C.H., 1992, Interfacial debonding and fiber pullout stresses of fiber-reinforced composites Part VII: Improved analysis for bonded interfaces, Mater. Sci. Eng., A154, $125-132$.

[25] Hsueh, C.H., 1995, A modified analysis for stress transfer in fiber-reinforced composites with bonded fiber ends, J. Mater. Sci., 30, 219-224.

[26] Hsueh, C.H., Becher, P.F., 1996, Residual thermal stresses in ceramic composites, Part II: with short fibers, Mater. Sci. Eng., A212, 29-35.

[27] Hsueh, C.H., Young, R.J., Yang, X., Becher, P.F., 1997, Stress transfer in a model composite containing a single embedded fiber, Acta. Mater., 45(4), 1469-1476.

[28] Li, Y., Langdon, T.G., 1997, An examination of creep data for an Al-Mg composite, Metall. Mater. Trans. A., 28 (5), 1271-1273.

[29] Gao, X.L., Li, K., 2005, A shear-lag for carbon nanotube-reinforced polymer composites, Int. J. Solids. Struct., 42, 1649-67.

[30] Jiang, Z., Liu, X., Zhang, H., Li, G., Lian, J., 2005, An analytical model for elastic stress field distribution in fibre composite with partially debonded interface, Compos. Sci. Technol., 65, 1176-1194.

[31] Lee, W.J., Son, J.H., Park, I.K., Park, Y.H., 2010, Direct numerical predictions for the steady-state creep deformation of extruded $\mathrm{SiCw} / \mathrm{Al} 6061$ composites using a representative volume element with random arrangement of whiskers, Comput. Mater. Sci., 48(4), 802-812. 